\title{
3-D modelling and 3-D printing in design and manufacturing of optical sensors
}

\author{
Vitaly Yu. Pavlov', Victor V. Valkov² \\ ${ }^{1}$ Candidate of Physical and Mathematical sciences, Associate Professor, Associate Professor of the Department 319, Moscow \\ Aviation Institute (National Research University), Russia \\ ${ }^{2}$ Master's student of the Department 319, Moscow Aviation Institute (National Research University), Russia
}

\begin{abstract}
The main purpose of the study is an optimization of design and manufacturing technologies in application to optical sensors, defining the phase composition of different liquids. The sensors use effects of phase contrast and speckle interferomerty so the demands to precision and stability of their optical path geometry are very high. Special attention is paid to chemical composition of filaments in order to avoid dissolution and deformation of 3-D printed parts under contact with aggressive solvents and liquids under pressure. The possibilities to combine different materials in one 3-D printed part has been also studied.A feasibility study of the applicability of 3-D modelling and 3-D printing for piece, small batch and mass production of optical sensors has been carried out.
\end{abstract}

Key words:3-D modelling, 3-D printing, optical sensor, liquid, phase composition, speckle-interferometer.

\section{INTRODUCTION}

In recent years, the availability of additive technologies has increased several times due to significant reduction in their cost. This allows us to use the capabilities of 3-D printing widely in almost all spheres of human activity. According to Sculpteo's "The state of 3-D PRINTING" [4], the use of 3-D printing of prototypes has nearly doubled during two years (2017-2018), and this trend continues nowadays. In addition, production and conceptualization with 3-D printing also hold a leading position, making the R\&D triumvirate at the top. Figure 1demonstrates the results of Sculpteo research in a graph showing the percentage of 3-D printing applications.

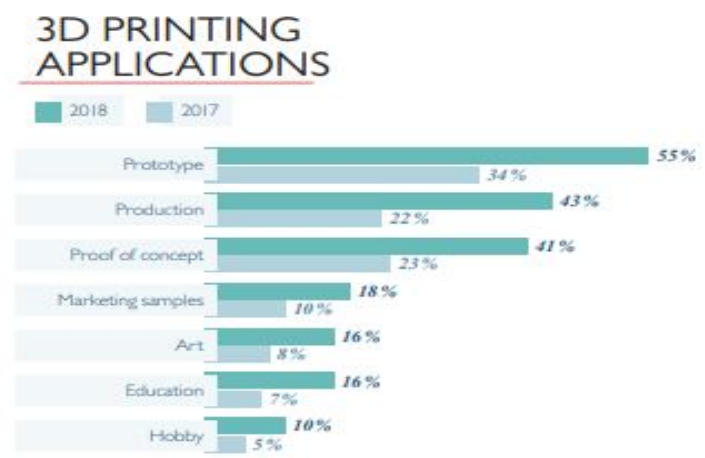

Figure 1: 3-D Printing Applications graph from the "the state of 3-D PRINTING" research
There are various ways to obtain a finished part using additive technologies,from the simplest and most affordable 3-D printers, even in the amateur segment, using the method of fused-deposition modelling (FDM) or photopolymerization of liquid monomer compositions (DLP or SLA) to more complex ones, including laser melting or sintering of materials (SLS), (SLM) or electron beam melting (EBM), which allows us to interact even with powders of refractory metals, such as titanium, to produce high-strength products for the aerospace industry.

The relevance of 3-D printing using FDM technology is confirmed by the relatively low cost of both 3-D printers and its consumables, a wide range of possible materials, a fairly high degree of printing accuracy and its high versatility.

These criteria are key for small-scale production for research purposes, since they allow to get parts of different shapes created from different materials using a single device as well as quickly repurpose production for other parts or make changes to existing ones in a short time. Research of the 3-D-printing market shown, that FDM is one of the most balanced at the aspect of cost-topossibilities in the starting segment of 3-D-printing technologies. The results of that research shown a good economical feasibility in accordance with an earlier research of the 3-D-printing market by another group of scientists, that was published in the Journal of International Business Studies [1].

Considering the economic feasibility of using 3-D printing for prototyping purposes for the needs of small laboratories or technology parks that cooperate with several laboratories (or other customers) at a time, universality is no less important criterion than the cost of materials or means of production. In the case of other plastic shaping technologies, such as vacuum forming or molding, it is necessary to produce unique casting masterform for each subsequent part, which are characterized by high production complexity and cost. In the case of using a 3-D printer, in fact, it is possible to produce almost the entire range of desired products on a single working surface using a single working tool, almost without resorting to the use of master forms or other auxiliary devices. 
Vitaly Yu. Pavlov et al., International Journal of Emerging Trends in Engineering Research, 8(6), June 2020,2496 - 2500

\section{MATERIALS AND METHODS}

\subsection{Selection of methods of production}

In order to effectively select solutions aimed at optimizing the production of optical fuel pollution sensor housings and body parts, it was first necessary to create a list of criteria for selecting the above-mentioned solutions. To do this, it was necessary to analyze the type, needs and nuances of production, its stage of development and trends.

At this period we need an experimental small-scale production serving the needs of the laboratory that develops and is engaged in the current revision of bench samples and prototypes of optical sensors. Thus, this production is characterized by:

- small need for the same type of parts. This requirement is derived from the small-scale experimental nature of the production of body parts and housings that meets the needs of a laboratory that is not currently conducting mass experiments with multiple samples at the same time;

- the need to change quickly the properties and characteristics of a particular part. This requirement comes from the fact that during the tests it may often be necessary to urgently refine or create an alternative drawing or model for which the part is made, according to the requirements of the experiments carried out;

- $\quad$ the need to quickly replace a damaged part during testing. This requirement comes from the fact that during the tests parts are subjected to various physical loads often exceeding the strength of the parts which leads to their damage and leads to the need for rapid replacement in order to avoid delays in the experimental development plan;

- $\quad$ highcomplexity of manufactured parts and cases assembled from them. This requirement comes from the fact that the final product is a complex device that requires simultaneous placement and fixation of several different sensors inside the housing, cable separation for them and the formation of a working zone while ensuring sufficient structural rigidity of the structure.

On this basis we can distinguish several requirements for a specific method of manufacturing cases and body parts of devices.

Technological requirement:

- low cost of manufacturing a single part;

- low cost of organization of parts manufacturing;

- high efficiency of using the material to make the part;

- $\quad$ production of parts in small batches;

- ability to manufacture complex parts;

- $\quad$ quick manufacture of parts;
- ability to reorient parts manufacturing to a different model;

- $\quad$ ability to change quickly the part manufacturing template (no dependence to molds and work pieces);

- the possibility of expanding the production of parts in the future.

Based on the above-mentioned requirements we can conclude that the introduction of 3-D printing using FDM technology was justified in view of meeting most of these requirements. This was followed by a study of specific ways TO implement 3-D printer kinematics in order to select the most appropriate technology at the initial stage of studying this technology. The Cartesian Robot kinematics was recognized as the most stable and easy-touse and maintain kinematics implementation, similar to the kinematics of classic CNC machinery. Within this family, there are different ways to use and group spatial axes, but they all share the same principle of the XYZ coordinate grid, which is represented in a scheme on the RepRap family page [2].

\subsection{Methods of production}

During theimplementation of 3-D printing in the design and prototyping process of optical sensors, it was decided to divide the application into three methods based on the required number and quality of parts which affects the specific application of 3-D printing technology:

- single pieces of parts. Such parts are body prototypes, demonstration models, additional equipment, and so on. These parts are required in small quantities at the level of single copies,sothey cfn be produced directly by 3-D printing at low speed and maximum print quality;

- small batches of parts. They are manufactured either entirely by 3-D printing, or are prototyped for subsequent production using classic means of production in a transitional multi-stage technological process;

- mass production of parts. 3-D printing is used to produce prototypes of molds and other auxiliary devices for further mass production.

Further, all three methods of using 3-D printing will be discussed in more detail with a description of the features and aspects of the application.

\subsubsection{Single pieces of parts}

In case of prototyping a piece of part that does not require mass production, it is advantageous to produce it using 3D printing directly, avoiding the unnecessary costs associated with ordering from commercial-oriented manufacturers who benefit from mass production in order to maximize their production capacity. When ordering such parts, manufacturers set requirements for ordering a certain number of samples, a fairly large batch. This is unprofitable both because of the high cost and the acquisition of unnecessary units of production, and 
because of the long wait for the order execution queue. Thus, it was decided to set up local in-lab production, since when purchasing an Amateur-level 3-D printer, the cost of buying it and putting it into operation is covered by savings on abandoning third-party manufacturers. Also, an important advantage is the flexibility of production, when getting an unsatisfactory result of experiments with an existing prototype of the sensor body, in less than a day it's possibleto make adjustments to the prototype model and create a new prototype, minimizing time costs and thus increasing the efficiency of the whole research process.

\subsubsection{Small batches of parts}

In the case of prototyping a small batch of parts, it was decided to use a multi-stage production process, in which 3-D printing acts as one of the fundamental stages that allow you to get the exact shapes of blanks for the production of final samples. 3-D printing is used to produce blanks for master forms. From these blanks, heatresistant gypsum moulds are made using wax casting, allowing small batches of parts to be produced using casting or vacuum forming with minimal costs, as well as avoiding the cost of mass production by a commerciallyoriented third-party manufacturer. Disadvantages of 3-D printing FDM technology, such as low temperature resistance, donot affect the finalsample of master form. Final master form is produced with more resistant materials using the 3-D printed sample, but there remain advantages of 3-D printing, such as automated production of starting sample with high precision and without the use of a direct labour of the laboratory employee, saving and efficient use of time.

\subsubsection{Mass production of parts}

In a situation where a mass production of parts is needed it still necessary to resort to help of third-party production manufacturers to produce master forms that allow production of several required parts at once. But even in this case 3-D printing brings its own contribution, as it allows creation of a prototype or mass-dimensional layout of the master form for a better understanding of what and how it should be made. Among other things, the combination of having a model of the desired part and a visual sample significantly speeds up negotiations and reaching an understanding with a third-party manufacturer regarding the production of the desired master forms. In some cases the production of a plastic prototype of the master form allows you to diagnose and correct defects in the 3-D model of the final master form at an early stage, which also indirectly allows you to avoid unnecessary costs for reproducing a new correct version of erroneous sample.

\subsection{Methods of modelling}

When considering the modelling technique it is necessary to take into account both the specifics of 3-D printing and the specifics of the prototyped optical sensors themselves.
Speaking about the specifics of 3-D printing it is necessary to keep in mind some of its technical features, first, the thickness of the thin-walled elements of the printed object. When designing parts it is necessary to take into account that the wall thickness should preferably have a multiplied value of the diameter of the 3-D printer extruder nozzle, which will allow printing the most well-sintered and durable elements and avoiding unnecessary voids between the printing lines. Secondly, it is necessary to consider that the deposit occurs in layers which means that in the case of the production of objects with overhanging structures (horizontal ledges, slopes at a certain angle, the arches, caps, etc.) it is necessary to consider the maximum possible angle for the formation of these structures with respect to the vertical part. This value depends on the specific material and ranges up to 60 degrees from the normal to the vertical surface of the part. A lower value corresponds to stable printing, and a higher value requires taking measures to support these elements or finding another way to position the model (a different base plane, rotation, etc.).

When considering the features of the optical sensor housings themselves, it is necessary to take into account the high requirements for the rigidity of the prototype design, which ensures the stability of the optical path in all modes of the sensor operation under pressure, mechanical stress and vibration. These requirements derive from the nature if speckle interferometry.[5]. For prototyping purposes it is impractical to use the creation of monolithic parts that are $100 \%$ filled with plastic in volume. To ensure the requirements that were mentioned before, it is necessary to resort to the use of an algorithm for filling the case in the form of a horizontal honeycomb, every few layers of printing overlapped with vertical solid layers. The formation of such a frame allows us to significantly increase the structural rigidity of the prototyped part relative to the hollow one, while significantly save material and reduce printing time relative to full filling. It is possible due to tweaking of printing parameters on a slicing stage, when the solid body model layered and turned into a chain of commands for 3-D printer. Combining different filling patterns and temperature settings it is possible to achieve light and well layersintered part. Screenshot of honeycomb filling visualization is shown on Figure 2 down below.

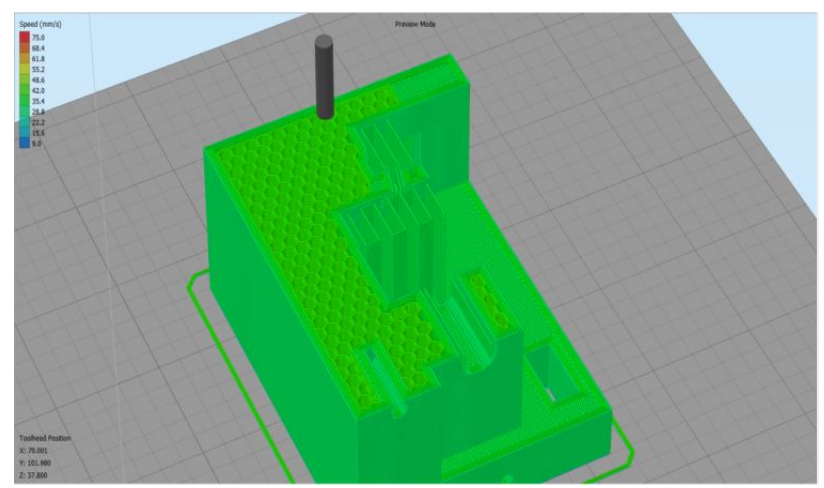

Figure 2: Honeycomb filling pattern visualization on a model slicing stage 
Vitaly Yu. Pavlov et al., International Journal of Emerging Trends in Engineering Research, 8(6), June 2020,2496 - 2500

\subsection{Materials}

Since the market analysis found that the FDM technology was sufficient for the prototyping goals we used 3-D printer that functioned on the basis of this technology.

This technology is characterized by sufficient economic accessibility and economy, while having a fairly wide versatility due to the materials used and the possibility of replacing the used printing material in a wide range, from the simplest plastics to composites with carbon fibre reinforcement.

As part of the analysis of available materials, the main criteria were the availability, sufficient structural strength and resistance to chemical effects of petrochemical group products used in the aviation industry, in particular-to aviation fuel. According to these criteria, PLA plastic is suitable because it has a good degree of resistance to various oils and fuels (according to GOST 12020-72, part weight change $<1 \%$ ). In addition the obvious advantage of this material is its high degree of stability, the absence of significant shrinkage when cooling, which allows us to produce 3-D printing without using an isolated thermal camera.

Furthermore, calculating the most effective heating temperature range for exact materials and usage of acquired measurements as printing temperature parameters will optimise the whole efficiency of 3-D printing process. Using the modified equation for calculating disk heating it is possible to obtain the most accurate temperature range to achieve uniform heating of the entire internal volume of the plastic rod passing through the heater without overheating of the outer bounds of plastic bar[6].

Later, in parallel with the development of a speckle interferometric sensor for quality control of aviation technical liquids, a study was started on the possibility of adapting this sensor to the needs of the chemical and biological industry in order to analyze the applicability of the sensor for monitoring the presence and condition of microorganisms in liquids. At the stage of testing the possibility of detecting these microorganisms when using already used electronic components of the sensor with minimal adjustments due to the software component of the analyzer, there was a need to conduct experiments with biologically active liquids containing both the microorganisms themselves and traces of their life activity. Because of this, ABS plastic has been used and is planned to be used in parallel for prototyping the sensor body since it is resistant to biological influences.

Speaking of other materials, SBS and PET were not used because most of them are partially transparent, which would create false signals in the output diagram of the optical sensor. Even the thick layers of materials still allow some light to pass through it, making it not suitable for experimental purposes with optic sensors, according to series of experiments [3], which were made by members of Russian 3-D-printing community.PETG usability is still being researched at the moment due to its stability and possible ABS-level structural strength and PLA-level stability, but higher material cost.

\subsection{Experiments and perspectives}

All the methods and materials mentioned above have been successfully tested and implemented in the prototyping process of the optoelectronic sensor housings being developed. At the moment, experiments are being conducted on new methods of applying 3-D printing in this particular area in two directions, as well as in the prospects for combining them.

\subsubsection{Combination of materials}

The first method is to combine several (two or more) printing materials, such as ABS+PLA, in order to combine their advantages and offset their disadvantages. An example of such levelling can be considered to increase the resistance to the actions of microorganisms of parts made of easy-to-process plastic PLA by covering it with protective layers of ABS plastic. Also, we consider the prospects for reinforcing ribs made of stronger and hightemperature-sintered ABS plastic housings from the PLA array in order to increase their structural strength to a greater extent than currently provided by the use of structural filling in the form of honeycombs with reinforcing overlaps.

Two main ways of doing this are considered. The first method is to use a more technically advanced 3-D printer capable of parallel printing with two extrusion modules, respectively, supporting two different temperature modes $\left(\sim 180^{\circ} \mathrm{C}\right.$ for $\mathrm{PLA}, \sim 240^{\circ} \mathrm{C}$ for $\left.\mathrm{ABS}\right)$. This method is positively characterized by the time spent on printing the combined part, but is quite expensive due to the cost of a 3-D printer with two extruders. The second method, an alternative to the first, is to use a single-extruder printer, which will replace the printing material used and the temperature mode. This method is much cheaper than purchasing a more technically complex 3-D printer, but it is characterized by high labor intensity and the need to optimize 3-D models of parts for printing in this way, up to the separation of a single part into several parts in order to simplify the imprinting of reinforcing elements.

\subsubsection{Encapsulation of materials}

The second experimental method of using 3-D printing is to encapsulate third-party parts in the printed prototype. Considering 3-D printing of a prototype optical cuvette, the question of ensuring the passage of a laser beam through it was raised. An obvious option was to use pasted inserts made of optical glass or quartz, but this solution is not reliable enough in the case of liquid analysis under pressure. Since the bonding takes place outside the finished part, the pressure directed from the inside on the walls can easily destroy the connection of the glass with the body of the cuvette. Encapsulation, along with sealing the joint, solves this problem. The essence of 
Vitaly Yu. Pavlov et al., International Journal of Emerging Trends in Engineering Research, 8(6), June 2020,2496 - 2500

encapsulation is to insert glass walls directly into the part during the printing process.

The problem with this method is that the material is deposited in layers, and the movement of the extruder on the layer is purely horizontal, which does not allow the 3D-printing method FDM to get the part with a pre-placed glass insert.

A comprehensive solution to this problem is software optimization of the 3-D printing process and optimization of the 3-D model. Pauses are inserted in the GCode of the printing process, allowing installing encapsulated elements in newly formed niches and slots right during printing.

\section{RESULTS}

In the course of the study the applicability of 3-D modeling and 3-D printing to optimize the development process, and prototyping speckle interferometric sensors was conducted the analysis of design and production, commissioning is carried out, commissioned FDM 3-D printer. Tests of the suitability of various plastics were carried out. This printer has been used for prototyping cases and other parts of the above-mentioned sensors. Work on the further development of these buildings, their improvement and refinement continues. Also, further prospects for the development of 3-D printing in the framework of the tasks and needs of the laboratory are outlined, a series of experiments with various methods of integrated use of 3-D printing, in multi-stage production, creating products with the encapsulation of third-party materials or combining and fusing different materials in one part in order to obtain new properties and characteristics.

\section{DISCUSSION}

Considering the results obtained, it is impossible not to note the high requirements for theoretical training and understanding of processes even when using even fairly simple 3-D printers working on FDM technology. The use of these printers required extensive analysis of both the material and technological base. However, after implementing this technology, a truly powerful and flexible tool was obtained for making prototypes and blanks of almost any parts required for the development of optical sensors.

The most interesting part of this study is the experimental work being carried out on the use of 3-D printing as an intermediate production link that facilitates the production of large batches of parts, as mentioned in paragraph 2.2, as well as a means of manufacturing structurally complex parts that include various materials or encapsulation of third-party objects. These experiments are considered promising and sufficiently interesting from the point of view of the novelty of combining production technologies.

In general, the introduction of 3-D modelling and 3-D printing in the prototype production of optical sensors is recognized as successful and sufficiently meets the requirements set at the beginning. This production has made it cheaper and faster to get prototypes of the required parts, which increases the overall efficiency of the laboratory.

\section{CONCLUSIONS}

The study provided a broad analysis of the 3-D printing industry, its current state and prospects. Goals for the introduction and effective use of 3-D printing for laboratory needs have been set and achieved. The solutions mentioned in the article for the use of PLA and ABS materials in production, together with the use of software correction algorithms for internal filling of parts, are considered optimal. The way of further research of 3-D printing applications in the future is outlined, and experiments have been started to assess the feasibility and effectiveness of these applications. The production of 3-Dprinted prototypes of molds for the multi-stage production process has been started. The collection of research data on the possibility and specific method of encapsulating objects in printed products has been started.

\section{REFERENCES}

1. A. Laplume, B. Petersen, J. Pearce. Global value chains from a 3-D printing perspective, in Journal of International Business Studies.2016, 47. 10.1057/jibs.2015.47.

2. RepRap.org, 2012. RepRap Family. http://reprap.org/wiki/RepRap_Family_Tree.

3. Russian 3-D-printing community, https://3dtoday.ru/.

4. Sculpteo, the state of 3-D PRINTING, 2018, https://www.sculpteo.com/media/ebook/State_of_3DP 2018.pdf.

5. V. G. Ageev, Yu. A. Kostikov, V. Yu. Pavlov.Measuring the characteristics of pollutant particles in liquids by speckle-interferometry, in International Journal of Mechanical and Production Engineering Research and Development, 2018, Special Issue, Jun, pp. 549-554.

6. Yu. A. Kostikov, A. M.Romanenkov.The Technology of Calculating the Optimal Modes of the Disk Heating (Ball), in Civil Engineering Journal, 2019, Vol. 5, No. 6.

https://doi.org/10.28991/cej-2019-03091340 